\title{
Annealing effects in high modulus polyethylene
}

\author{
D. Göritz, J. Kemmerer, S. Kreitmeier, and R. Rossbacher
}

Universität Regensburg, Naturwissenschaftliche Fakultät II-Physik

\begin{abstract}
Summary: Ultra-high modulus polyethylene has a fibrillar structure in which an individual fibril is formed from a stack of ribbonshaped units. In contrast to the behaviour of high-pressure crystallised polyethylene on annealing, lamellae grow from the edges of the fibrils perpendicular to the draw direction. The thickness of the lamellae is less than $40 \mathrm{~nm}$. The macrofibrils are partially transformed as a function of annealing time into perpendicularly oriented lamellae.
\end{abstract}

Key words: Polyethylene, high modulus, annealing, fibril, lamella.

\section{Introduction}

The elastic moduls of ultra-highly oriented polymers is a function of the degree of deformation, if the high orientation is produced by drawing or by solidstate extrusion [1]. The properties controlled by the deformation should therefore react sensitively to raising the temperature or to heat treatment, since for these high orientations entropy-controlled restoring forces will have a strong influence on morphological changes which occur during annealing. It is well known that on annealing ultra-high modulus polyethylene the long period increases with temperature but that the average crystal thickness in the direction of the chain axis decreases. This was attributed to a crystallisation of chains originating from fibrillar regions into lamellar-type crystals [2]. The purpose of this work was to follow the structural changes which occur on annealing by means of electron microscopy.

\section{Experimental}

The highly oriented sheet samples of high density polyethylene (Rigidex 50, $M_{w} \approx 10^{5}$ ) were produced by Ward [1] by drawing at $75^{\circ} \mathrm{C}$ to draw ratios $\lambda$ of 11 . Specimens were annealed for various times at $120^{\circ} \mathrm{C}$ either with fixed or with free ends.

Specimens were investigated by scanning electron microscopy (SEM) (Philips PSEM 500). The specimens were not specially treated for the SEM investigations. They were only sputtered with gold to avoid electrostatic charging. Differential scanning calorimetry (DSC) was carried out using a Perkin Elmer DSC II calorimeter, in which all the specimens were heated at $5 \mathrm{~K} / \mathrm{min}$ at constant length.

\section{Results}

\subsection{Fibrillar structure}

As shown by Owen [3] for the case of cold-drawn HDPE samples with rectangular cross-section, one also finds for the high modulus drawn sample of draw ratio $\lambda=11$ that the deformation proceeds anisotropically in a plane perpendicular to the draw direction.

SEM micrographs of the broader surface of the ribbon-shaped specimen (fig. 1a) and of the edge (fig. 1b) show the well-known fibrillar structure, in which the width of the fibrils is considerably smaller on the edge than on the face.

One sees from figure $1 \mathrm{a}$ that the macroscopic fibrils consist of stacks of ribbon-shaped subunits which reflect the dimensions of the specimen prior to drawing. The different dimensions of the fibrils was attributed by Owen [3] to different deformation ratios perpendicular to the draw direction, which led to a preferred orientation of inter-microfibrillar tie molecules in the plane of the sheet.

\subsection{Annealing effects}

According to the model of Gibson et al. [4], the crystalline regions in the fibrils of ultra-high modulus polymers consist of fully extended chains. One would therefore expect that on annealing ultra-high modulus polymers an epitaxial growth of chain-folded lamellae 


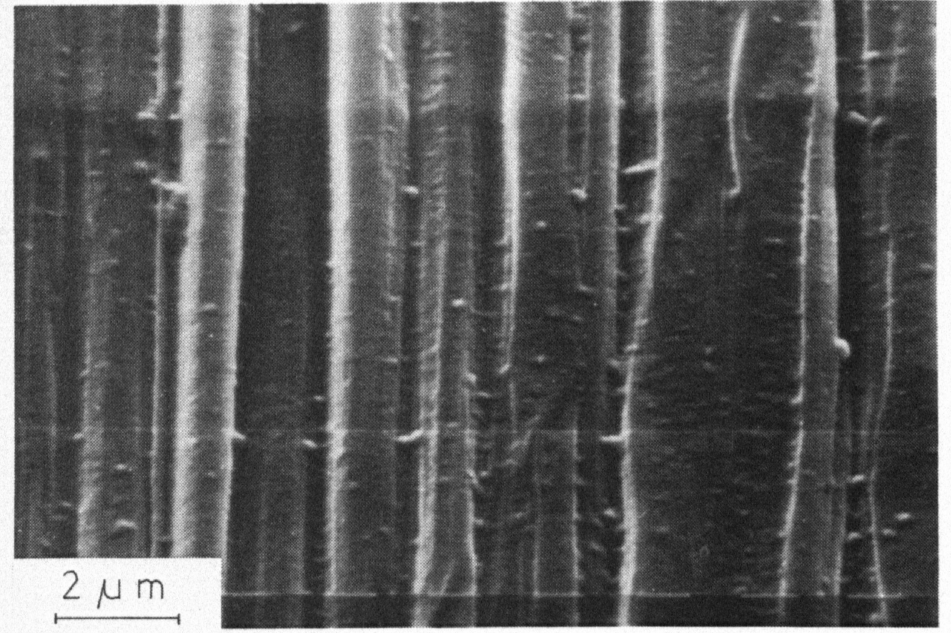

a

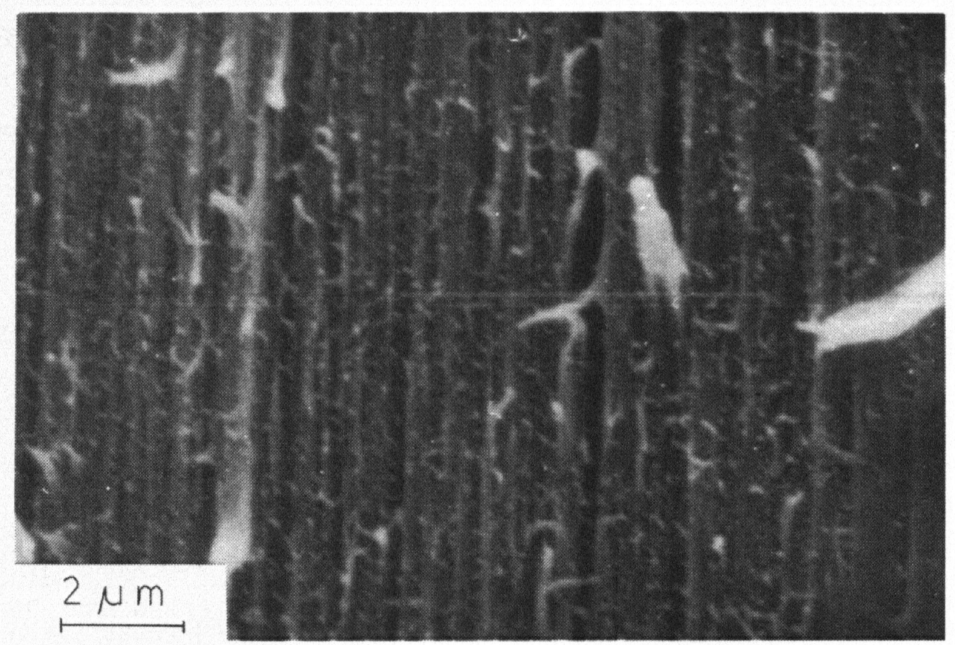

b

Fig. 1. Scanning electron micrographs of HDPE samples drawn to $\lambda=11$. View of face (1a) and edge (1b) of specimen with rectangular cross-section

would occur, as is the case for pressure-crystallised polymers containing extended-chain crystals.

Figure 2 shows SEM micrographs of specimens annealed for various times at $120^{\circ} \mathrm{C}$. Whereas in highpressure crystallised material under the same annealing conditions lamellae completely covering the extended-chain crystals have already formed after 3 minutes [5], one sees here from figures $2 a-2 d$ that a strongly time-dependent transformation of macrofibrils into lamellae at right-angles to them occurs. Since no fine lamellar overgrowth appears on the untransformed macrofibrils even after 30 minutes annealing time, it appears that an analogous surface melting cannot be responsible for the mechanism. Figures $2 a$ and $2 b$ suggest much more that the ribbon-shaped elements of the macrofibrils melt from their edges and with increasing annealing time grow perpendicularly to the original orientation direction.
When growing lamellae meet between the macrofibrils they coalesce, thereby lowering the surface energy. After an annealing time of 60 minutes the macrofibrils have disappeared. Their previous position is however still discernable through a raised packing density of lamellae (2a, 2d).
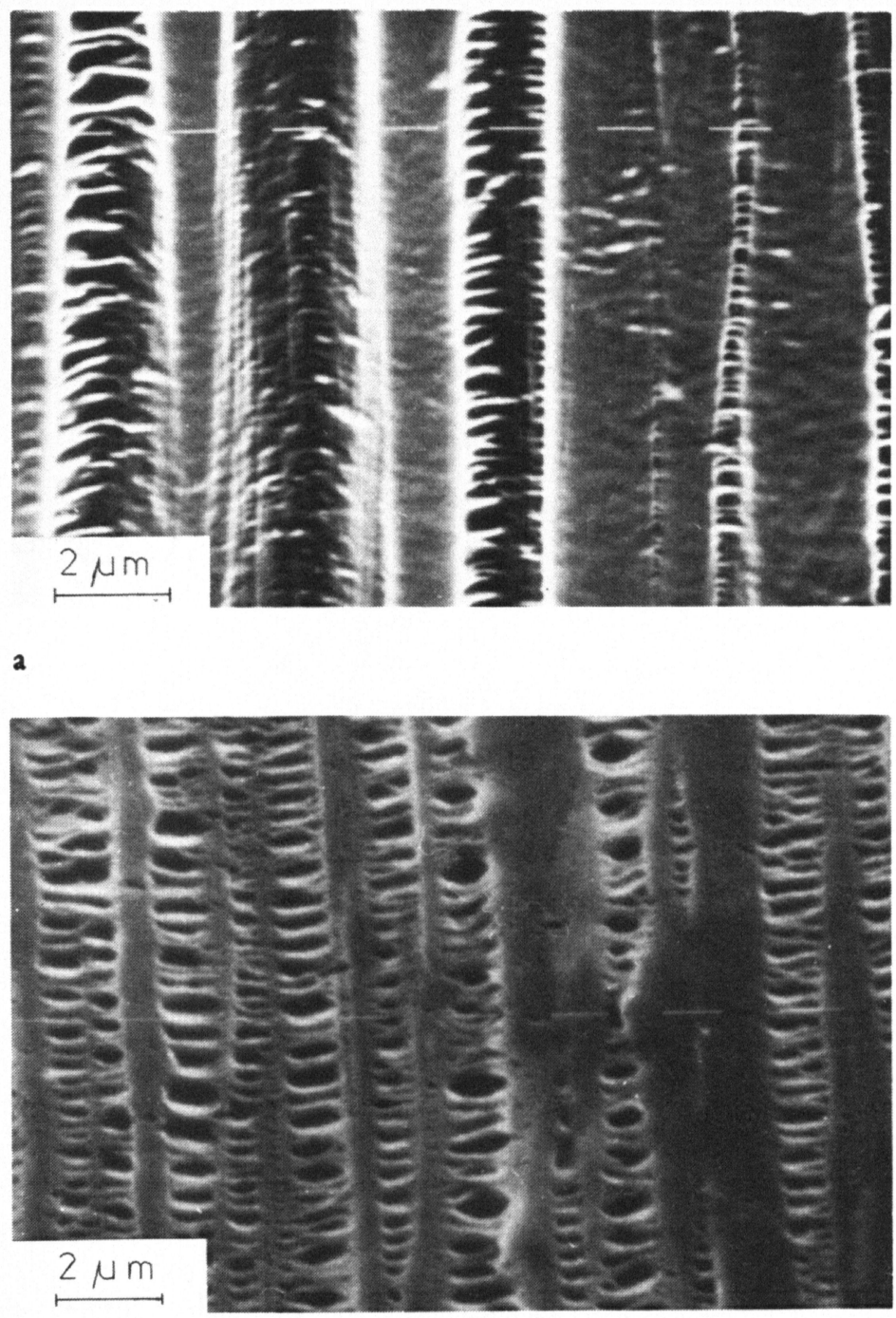

b

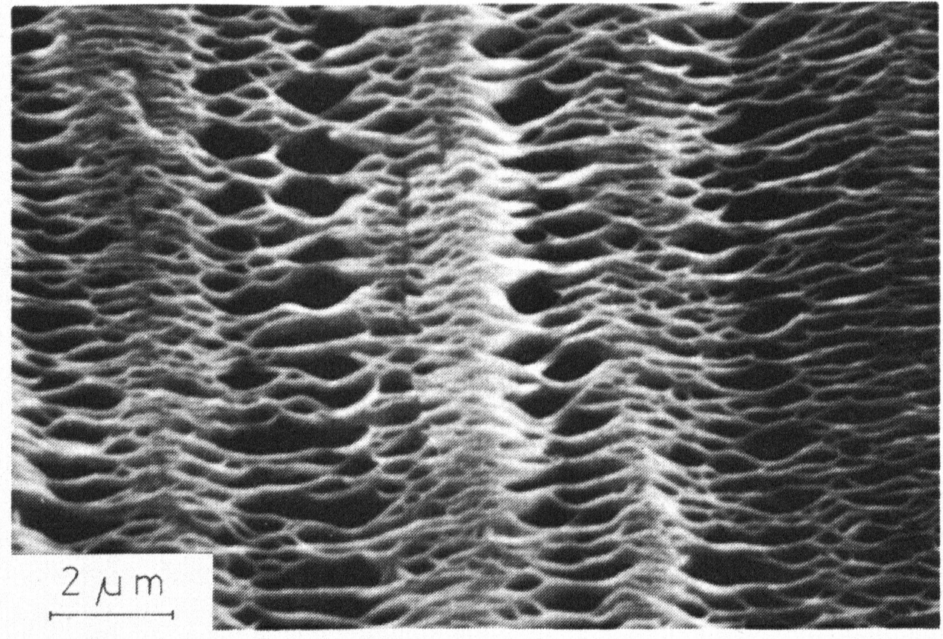




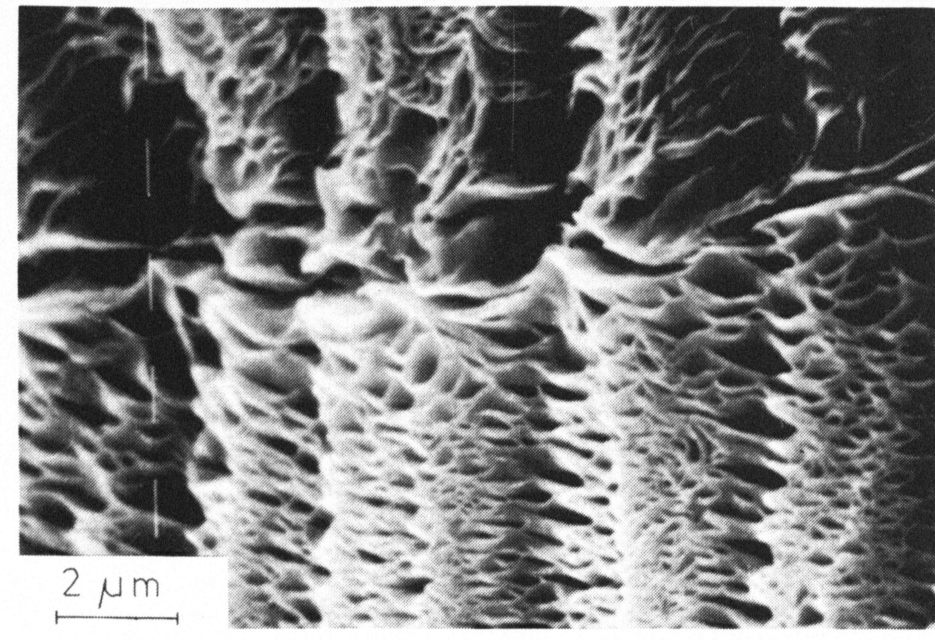

d

Fig. 2. Scanning electron micrographs of the same sample as in figure 1 after annealing at $T_{a}=120^{\circ} \mathrm{C}$ (a) 3 mins., (b) 30 mins. (c) 60 mins., (d) 60 mins. In figures $2 a-c$ the specimens were annealed with fixed ends, in figure $2 \mathrm{~d}$ with free ends

Figure 3 at higher magnification shows these densely packed units, each with a thickness of approximately $40 \mathrm{~nm}$, in place of the old macrofibrils. If these lamellae leave the region of the previous macrofibril, typically 3-5 units coalesce into one lamellar bundle. The larger the number of units which form a bundle, the more structureless is its surface. The chain direction within the lamellae is at present unknown, but its determination is the aim of present work.

Figures $2 \mathrm{c}$ and $2 \mathrm{~d}$ show specimens which had been annealed for 60 minutes, one with free and the other with fixed ends. In both cases the macrofibrils have transformed partially into lamellae. In the latter case one notices however that on annealing with fixed ends a more uniform structure occurs.

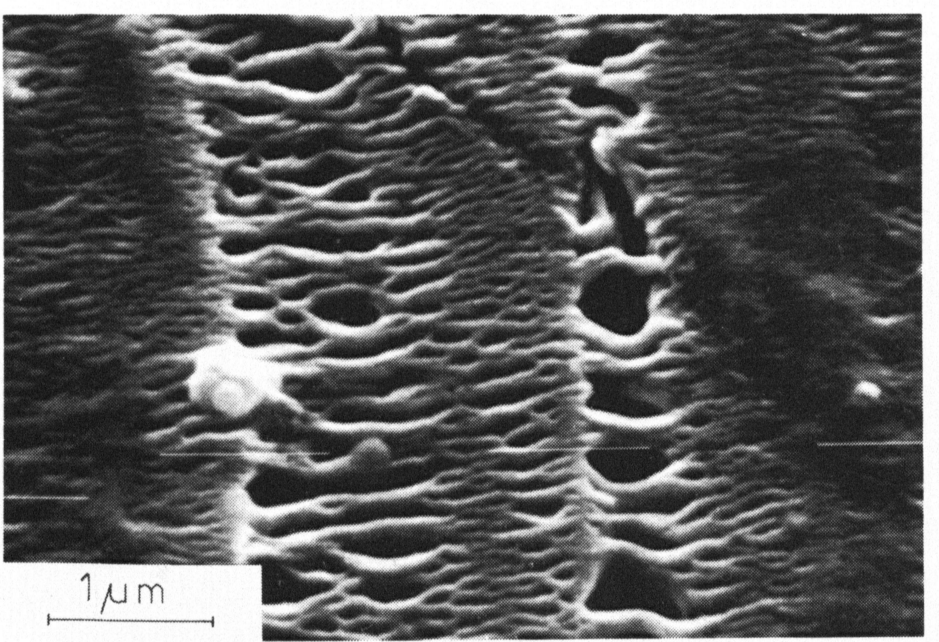

Fig. 3. Scanning electron micrograph of the specimen in figure $2 \mathrm{c}$ at higher magnification
DSC measurements of the melting curves for specimens held at constant length led to the surprising result that the annealed specimen, i. e. after the transformation of macrofibrils into lamellae, showed a melting region shifted to higher temperatures (fig. 4). The melting behaviour of the annealed specimens, which possessed an elastic modulus only $10 \%$ smaller after the transformation, cannot therefore be compared with that of isotropic material with a lamellar structure. Assuming the melting enthalpy to be constant, then an explanation for the occurence of a melting region shifted to higher temperatures requires that the noncrystalline environment of the crystallites becomes more highly oriented on annealing. It is assumed that in the original state in the macrofibrils crystalline and amorphous regions lie parallel to eachother. On annealing, these amorphous regions readjust themselves such that they crystallise epitaxially onto the crystallites which are still present within the macrofibrils. If this material is melted, chains from the crystalline regions find a melt which is more highly oriented than before the annealing process. The melting temperature is therefore raised.

If, however, we do not assume that the melting enthalpy is independent of the deformation, then one departs from an entropy-controlled raising of the melting point due to annealing to one which is energycontrolled.

As a result of drawing at elevated temperature the crystallites become oriented with their $c$-axes in the draw direction and the macroscopic sample has a negative linear coefficient of expansion. Assuming that the top and bottom surfaces of the crystals do not consist entirely of chain folds, then the oriented

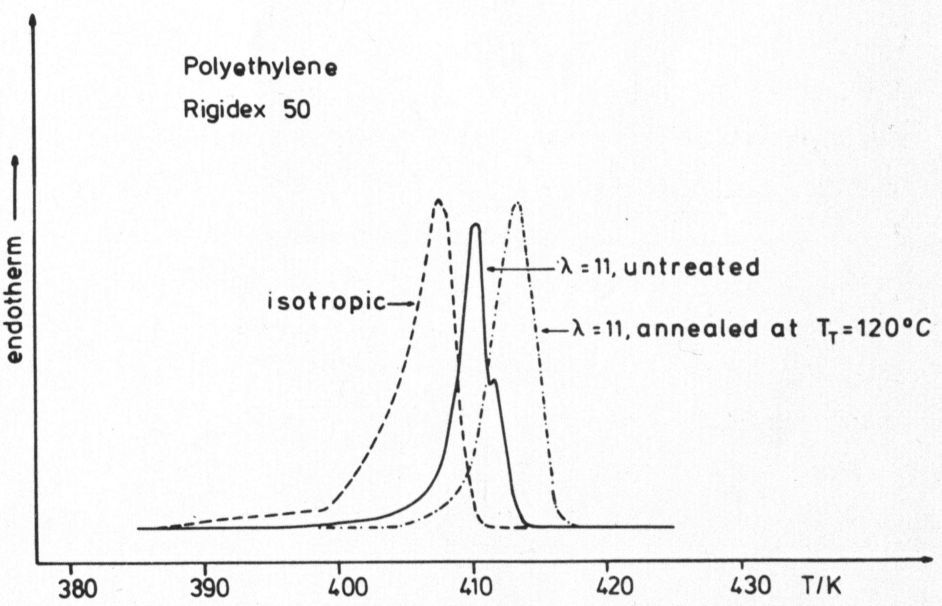

Fig. 4. DSC run of HDPE sample drawn to $\lambda=11$. Secimens had been previously annealed with fixed ends at $120^{\circ} \mathrm{C}$. The DSC measurement also took place with fixed specimen ends 
amorphous chains on these crystal surfaces experience a mechanical tension. Such a surface force mechanism, which is dependent on the mechanical deformation, would lower the melting enthalpy and lead to a lowering of the melting point. Due to annealing the mechanical tensions are lowered whereas a raising of the melting point is oberved (fig. 4).

Clements and coworkers [2] found from the linebroadening of the (002) reflection that the average crystal thickness in the chain direction decreased from 46 to $40 \mathrm{~nm}$ on annealing. This can be explained by the thickness of the elementary lamellae of approx. $40 \mathrm{~nm}$ shifting the average size of the crystallites to lower values, if it is assumed that the chains in the elementary lamellae lie parallel to the initial draw direction.

\section{References}

1. Capaccio, G., I. M. Ward, Nature (Phys. Sci.) 243, 143 (1973).

2. Clements, J., R. Jakeways, I. M. Ward, G. W. Longman, Polymer 20, 295 (1979).

3. Owen, A. J., Colloid \& Polymer Sci. 259, 252 (1981).

4. Gibson, A. G., G. R. Davies, I. M. Ward, Polymer 19, 683 (1978).

5. Wunderlich, B., L. Mellilo, C. M. Cormier, T. Davidson, G. Snyder, J. Macromol. B3, 485 (1967).

Authors' address :

Received March 7, 1983; accepted April 19, 1983

D. Göritz

Naturwissenschaftliche Fakultät II-Physik

Universität Regensburg

Universitätsstr. 31

8400 Regensburg 